BULL. AUSTRAL. MATH. SOC.

$58 \mathrm{C} 15,58 \mathrm{C} 20,58 \mathrm{G99}$

VOL. 27 (1983), 381-394.

\title{
AN INVERSE MAPPING THEOREM FOR SOBOLEV CHAINS AND ITS APPLICATION
}

\author{
Truong Công NGHÊ
}

\begin{abstract}
The author combines the methods used by Yamamuro and Omori to define a differentiation in Sobolev chains and obtain an Inverse Mapping Theorem. He then uses this theorem to give a new proof for a result of Sunada on the local finite-dimensionality of the solution space of a non-linear elliptic differential operator with smooth coefficients.
\end{abstract}

The purpose of this paper is to combine the methods used by Yamamuro [7] and Omori [3] to define a differentiation in Sobolev chains and obtain an Inverse Mapping Theorem. Here, for simplicity, we only consider the differentiability of class $C^{l}$ even though the theorem can be extended to the class $c^{k}$ for any integer $k \geq 1$ and to the class $C^{\infty}$. As its first application we give a new proof for a result of Sunada [6] on the local finite-dimensionality of the solution space of a non linear elliptic differential operator with smooth coefficients.

The paper consists of three sections. In the first section we define regular $c_{B \Gamma}^{l}$ maps between Sobolev chains and give a sufficient condition for a map to be a regular $c_{B \Gamma}^{l}$ map. The next section is for stating and proving the Inverse Mapping Theorem and the last section is for its application.

Received 26 January 1983. The author wishes to thank Sadayuki Yamamuro for his' encouragement and helpful discussions. 


\section{Regular $c_{\beta \Gamma}^{l}$ mappings}

Recall that a Sobolev chain [3], [8] is a sequence $\left\{E, E^{i}: i \geq d\right\}$ ( $d$ being a positive integer) satisfying:

(i) each $E^{i}$ is a Banach (or Hilbert) space with the Banach (or Hilbert) norm $|\cdot|_{i}$,

(ii) for each $i \geq d, E^{i} \supseteq E^{i+1}$ and $|\cdot|_{i} \leq|\cdot|_{i+1}$ on $E^{i+1}$

(iii) $E$ is the intersection of all $E^{i}$ and has the inverse limit topology defined by the $E^{i}$ 's,

(iv) $E$ is dense in every $E^{i}, i \geq d$.

Thus, since $\left\{|\cdot|_{i}: i \geq d\right\}$ is an (increasing) sequence of norms, $E$ has the structure of a Fréchet space. In practice, Sobolev chains often arise in the following way. Let $E$ be a Fréchet space defined by an increasing sequence of norms $\left\{|\cdot|_{i}: i \geq d\right\}$ which are pairwise coordinated [8, p. 337]. Then $E$ can be considered as the limit space of the Sobolev chain $\left\{E, E^{i}: i \geq d\right\}$ where, for each $i, E^{i}$ is the completion of the normed space $E_{i}=\left(E,|\cdot|_{i}\right)$.

Now let $\left\{E, E^{i}: i \geq d\right\}$ and $\left\{F, E^{i}: i \geq d\right\}$ be two Sobolev chains. Let $U \subseteq E$ be open and $\Phi: U \subseteq E \rightarrow F$ be a map. We may consider the $\Gamma$-differentiability of $\Phi$ by taking the following natural calibration [7] for $(E, E)$ :

$$
\Gamma=\left\{\left(|\cdot|_{i},|\cdot|_{i}\right): i \geq d\right\}
$$

Then, by a result in [7], for any integer $r \geq 0, \Phi$ is of class $c_{\Gamma}^{r}$ if and only if $\Phi: U \subseteq E_{i} \rightarrow F_{i}$ is $c^{r}$ (for all $i \geq d$ ) in the usual sense of mapping between normed spaces.

Let $A: E \rightarrow E$ be linear $\Gamma$-continuous. We say that $A$ is quasi $B \Gamma$-continuous or is $B \Gamma$-continuous if there exist constants $C>0$ 
(independent of $i$ ) and $D_{i}>0$ such that

$$
|A(u)|_{i} \leq c|u|_{i}+D_{i}|u|_{i-1} \text { for all } u \in E \text { and } i \geq d+1 \text {. }
$$

We denote by $L_{B \Gamma}(E, F)$ the space of all quasi $B \Gamma$-continuous linear maps $E \rightarrow F$. Then, as vector spaces, we have ([7])

$$
L_{B \Gamma}(E, F) \subseteq L_{B \Gamma}(E, F) \subseteq L_{\Gamma}(E, F) \subseteq L(E, F)
$$

If $A \in L_{B \Gamma}(E, F)$, let $|A|$ be the Omori semi-norm of $A$ defined in [3], p. 141,

$$
|A|=\inf \{C: C \text { is a possible constant in (2)\}, }
$$

and, for each $i \geq d$, define the following norm on $L_{\beta \Gamma}(E, F)$ :

$$
\|A\|_{i}=\max \left(|A|,\|A\|_{i}\right) \text { for } A \in L_{\beta \Gamma}(E, F) \text {, }
$$

where \|\|$A \|_{i}$ is the operator norm of $A$.

We endow $L_{\beta \Gamma}(E, F)$ with the canonical calibration $\left\{\|\cdot\|_{i}: i \geq d\right\}$. Note that any $A \in L_{B \Gamma}(E, F)$ can be extended to a linear continuous map $E^{i} \rightarrow F^{i}$ for all $i \geq d$.

We denote by $\mathrm{GL}_{\beta \Gamma}(E, F)$ the totality of elements $A \in L_{\beta \Gamma}(E, F)$ such that $A^{-1}$ exists and is contained in $L_{B \Gamma}(E, E)$.

(1.1). $\mathrm{GL}_{B \Gamma}(E, F)$ is open in $L_{\beta \Gamma}(E, F)$ endowed with the calibration $\left\{\|\cdot\|_{i}: i \geq d\right\}$ defined by (5).

Proof. This follows from [3, Theorem 11.1.2].

Now let $\Phi: U \subseteq E \rightarrow F$ be as above and let $a \in U$. We say that $\Phi$ is quasi $B \Gamma$-differentiable at $a$ (or $B \Gamma$-differentiable at $a$ ) if there exists an element $A \in L_{B \Gamma}(E, F)$ such that the following condition is satisfied: for all $i \geq d$, all $\varepsilon>0$, there exists $\delta>0$ such that

$$
|\Phi(a+v)-\Phi(a)-A(v)|_{i}<\varepsilon|v|_{i}
$$

whenever $|v|_{i}<\delta$ and $a+v \in U$. 
The map $A=D \Phi(a) \in L_{B \Gamma}(E, F)$ is then uniquely determined and is called the $\beta \Gamma$-derivative of $\Phi$ at $a \in U$. Thus we can define the notion of $\beta \Gamma$-differentiable map on $U$. We say that $\Phi$ is continuously $B \Gamma$ differentiable at $a \in U$ (or $\Phi$ is $C_{B \Gamma}^{I}$ at $a$ ) if $\Phi$ is $В \Gamma-$ differentiable in a neighbourhood of $a$ and the derived map $D \Phi: U \subseteq E \rightarrow L_{B \Gamma}(E, F)$ is $\hat{\Gamma}$-continuous at $a$ with respect to the natural calibration $\hat{\Gamma}=\left\{\left(|\cdot|_{i},\|\cdot\|_{i}\right\}: i \geq d\right\}$ for $\left(E, L_{B \Gamma}(E, F)\right)$. The notion of a $C_{\beta \Gamma}^{I}$ map on $U$ is then defined as usual. The notion of $C_{\beta \Gamma}^{p}$ maps $(r \geq 2$ or $+\infty)$ can also be defined. But here we are more interested in $C_{B \Gamma}^{7}$ maps.

A $C_{B \Gamma}^{l}$ map $\Phi: U \subseteq E \rightarrow F$ is called regular if and only if the following conditions are satisfied:

(i) there exists an open set $\Omega \subseteq E^{d}$ such that $U=\Omega \cap E$;

(ii) for each $i \geq d, \Phi$ can be extended to a $c^{1}$-map $\Omega \cap E^{i} \rightarrow E^{i}$.

The following proposition will give us a sufficient condition for a map to be a regular $c_{B \Gamma}^{l}$ map.

(1.2). Let $\left\{E, E^{i}: i \geq d\right\},\left\{F, F^{i}: i \geq d\right\}$ be two sobolev chains, $U \subseteq E$ be open such that $U=\Omega \cap E$ where $\Omega$ is an open convex set in $E^{d}$. Let $\Phi: U \subseteq E \rightarrow F$ be a map and endow $(E, F)$ with the calibration $\Gamma=\left\{\left(|\cdot|_{i},|\cdot|_{i}\right): i \geq d\right\}$.

Suppose that $\Phi: U \subseteq E \rightarrow F$ is of class $C_{\Gamma}^{2}$ [7], and satisfies the following condition: for alz $u \in U=\Omega \cap E, a l z v, v_{1}, v_{2} \in E$ and all $i \geq d+1$, we have

$$
|D \Phi(u) v|_{i} \leq c\left\{|u|_{i}|v|_{d^{+}}|v|_{i}\right\}+P_{i}\left(|u|_{i-1}\right)|v|_{i-1},
$$




$$
\text { (ii) } \begin{aligned}
\left|D^{2} \Phi(u) v_{1} v_{2}\right|_{i} \leq c\left\{|u|_{i}\left|v_{1}\right|_{d}\left|v_{2}\right|_{d}\right. & \left.\left|v_{1}\right|{ }_{i}\left|v_{2}\right|_{d}+\left|v_{1}\right|{ }_{d}\left|v_{2}\right|_{i}\right\} \\
& +P_{i}\left(|u|_{i-1}\right)\left|v_{1}\right|_{i-1}\left|v_{2}\right|_{i-1},
\end{aligned}
$$

where $C$ is a positive constant (independent of $i$ ) and $P_{i}$ is a polynomial with positive coefficients depending on $i$.

Then $\Phi: U \subseteq E \rightarrow F$ is a regular $c_{\beta \tilde{\Gamma}}^{1}$ map with respect to the calibration $\tilde{\Gamma}=\left\{\left(\left.|\cdot|\right|_{i},|\cdot|_{i}\right): i \geq d+1\right\}$.

Proof. Since $\Phi: \Omega \cap E \subseteq E \rightarrow E$ is of class $C_{\Gamma}^{1}$, for all $i \geq d$, we have, by [7],

$$
\Phi: \Omega \cap E \subseteq E_{i} \rightarrow F_{i} \text { is of class } C^{l}
$$

where $E_{i}=\left(E,\left.|\cdot|\right|_{i}\right)$ and $F_{i}=\left(E,\left.|\cdot|\right|_{i}\right)$.

We first prove that $\Phi$ is of class $c_{B \Gamma}^{l}$. Since, by (i), $D \Phi(u) \in L_{B \Gamma}(E, F)$ for all $u \in U$, it suffices to show that $D \Phi: U \subseteq E \rightarrow L_{\beta \Gamma}(E, F)$ is $\hat{\Gamma}$-continuous .

Now, for $u, u_{0} \in U$, we have

$$
\left|\left(D \Phi(u)-D \Phi\left(u_{0}\right)\right) v\right|_{i} \leq \int_{0}^{1}\left|D^{2} \Phi\left[u_{0}+t\left(u-u_{0}\right)\right] \cdot\left(u-u_{0}\right) \cdot v\right|_{i} d t
$$

Thus, by a simple calculation, using ( $i i)$, we have, for all $i \geq d+1$,

$$
\left|\left(D \Phi(u)-D \Phi\left(u_{0}\right)\right) v\right|_{i} \leq c\left|u-u_{0}\right|_{d}|v|_{i}+\tilde{P}_{i}\left(\left|u_{0}\right|_{i},\left|u-u_{0}\right|_{i}\right)|v|_{i-1},
$$

where $\tilde{P}_{i}$ is a polynomial in $\left|u_{0}\right|_{i}$ and $\left|u-u_{0}\right|_{i}$ with positive coefficients depending on $i$.

Thus, by definition of the Omori semi-norm,

$$
\left|D \Phi(u)-D \Phi\left(u_{0}\right)\right| \leq c\left|u-u_{0}\right|_{d} \leq c\left|u-u_{0}\right|_{i} \text { for all } i \geq d .
$$

From this it follows quickly that $D \Phi$ is $\hat{\Gamma}$-continuous.

To see that, for all $i \geq d+1, \Phi$ can be extended to a $c^{l}$ map from 
$\Omega \cap E^{i}$ to $F^{i}$, we notice that conditions (i) and (ii) in (1.2) ensure that, for all $i \geq d+1$, all $u \in \Omega \cap E$, all $v, v_{1}$ and $v_{2} \in E$, we have

$$
|D \Phi(u) v|_{i} \leq \hat{P}_{i}\left(|u|_{i}\right)|v|_{i} \text {, }
$$

and

$$
\left|D^{2} \Phi(u) v_{1} v_{2}\right|_{i} \leq \check{\vec{p}}_{i}\left(|u|_{i}\right)\left|v_{1}\right|_{i}\left|v_{2}\right|_{i}
$$

where $\hat{P}_{i}$ and $\check{P}_{i}$ are polynomials with positive coefficients depending on $i$.

Now, using (11), it can be seen that, for all $i \geq d+1$, $\Phi: \Omega \cap E \subseteq E \rightarrow F$ can be extended to a continuous map $\Omega \cap E^{i} \rightarrow F^{i}$ which is still denoted by $\Phi$.

Let $a$ be an arbitrary element in $\Omega \cap E^{i}$ and let $\left\{a_{n}\right\} \subseteq \Omega \cap E$ converging to $a$ in $E^{i}$. Then, by (7), for every $n$, the derivative $D \Phi\left(a_{n}\right) \in L\left(E_{i}, F_{i}\right)$ exists. We may consider it as a linear continuous map $E_{i} \rightarrow F^{i}$. Then it extends to a unique element $\overparen{D \Phi\left(a_{n}\right)} \in L\left(E^{i}, F^{i}\right)$ with the same norm. From (12) it can be seen that $\left\{d \Phi\left(a_{n}\right)\right\}$ is a cauchy sequence in $L\left(E^{i}, F^{i}\right)$ and thus converges to an element $D \Phi(a) \in L\left(E^{i}, F^{i}\right)$. Then it is easily seen that the map

$$
D \Phi: \Omega \cap E^{i} \subseteq E^{i} \rightarrow L\left(E^{i}, F^{i}\right): a \mapsto D \Phi(a)
$$

is continuous and the extension $\Phi: \Omega \cap E^{i} \subseteq E^{i} \rightarrow E^{i}$ is differentiable at $a$ with $D \Phi(a)$ as its derivative.

REMARK 1. The above proof shows that the regularity condition is a consequence of the inequalities $(i)$ and $(i i)$ in (1.2).

REMARK 2. (1.2) shows that all $C^{\infty} I L B-C^{2}$ normal mappings in [3] are regular $C_{B \Gamma}^{l}$ maps with respect to natural calibrations. 


\section{The Inverse Mapping Theorem}

Since, by (1.1), $\mathrm{GL}_{\beta \Gamma}(E, F)$ is open in $L_{B \Gamma}(E, F)$, we may expect that the Inverse Mapping Theorem is true for $C_{\beta \Gamma}^{1}$ maps between Sobolev chains. In fact, we have

(2.1). Let $\left\{E, E^{i}: i \geq d\right\},\left\{F, F^{i}: i \geq d\right\}$ be two Sobolev chains, $\Phi: U=\Omega \cap E \subseteq E \rightarrow F$ be a regular $C_{B \Gamma}^{1}$ map, where $\Omega$ is open convex in $E^{d}$. Suppose that $0 \in U$ and $\Phi(0)=0$ and assume that $D \Phi(0) \in \mathrm{GL}_{\beta \Gamma}(E, F)$.

Then $\Phi$ is a local $C_{\beta \Gamma}^{1}$-diffeomorphism at 0 (that is, there are open neighbourhoods. $\tilde{W}, \tilde{W}^{\prime}$ of 0 in $E, F$ respectively such that $\Phi: \tilde{W} \rightarrow \tilde{W}^{\prime}$ is a $C_{B \Gamma}^{\perp}-d i$ ffeomorphism).

Proof. This is basically the proof given by Omori [3] with suitable modification.

By hypothesis, $D \Phi(0): E \rightarrow F$ is a toplinear isomorphism satisfying, for all $v \in E$ and all $i \geq d+1$,

$$
\begin{aligned}
& |D \Phi(0) v|_{i} \leq \alpha|v|_{i}+\beta_{i}|v|_{i-1}, \\
& |D \Phi(0) v|_{i} \geq \gamma|v|_{i}-\delta_{i}|v|_{i-1},
\end{aligned}
$$

where $\alpha, \gamma, \beta_{i}, \delta_{i}$ are positive constants $(\alpha, \gamma$ being independent of $i$, and, for all $i \geq d, \Phi$ extends to a $c^{l}$ map

$$
\Phi: \Omega \cap E^{i} \subseteq E^{i} \rightarrow E^{i}
$$

Since $E$ (respectively $F$ ) is dense in every $E^{i}$ (respectively $\left.F^{i}\right), D \Phi(0)$ extends to a toplinear isomorphism of $E^{i}$ and $F^{i}$ for every $i \geq d$. We still denote the extensions by $D \Phi(0)$ which satisfy (13) and (14) for all $v \in E^{i}$ and all $i \geq d+1$.

Since $D \Phi: \Omega \cap E \subseteq E \rightarrow L_{\beta \Gamma}(E, F)$ is $\hat{\Gamma}$-continuous, there is $\delta^{\prime}>0$ such that 


$$
|u|_{d}<\delta^{\prime} \text { implies }|D \Phi(u)-D \Phi(0)|<\gamma / 3
$$

Thus, for all $i \geq d+1$, we have

$$
|u|_{d}<\delta^{\prime} \text { implies }|D \Phi(u) v-D \Phi(0) v|_{i} \leq(\gamma / 3)|v|_{i}+D_{i}|v|_{i-1} \text {. }
$$

Hence, from (14), for all $|u|_{d}<\delta^{\prime}$ and all $i \geq d+1$, we have

$$
|D \Phi(u) v|_{i} \geq(2 \gamma / 3)|v|_{i}-D_{i}^{\prime}|v|_{i-1} \text { for all } v \in E^{i} \text {, }
$$

where $D_{i}^{\prime}=\delta_{i}+D_{i}$.

Furthermore, since $\Phi: \Omega \subseteq E^{d} \rightarrow F^{d}$ is $C^{\perp}$ and $D \Phi(0): E^{d} \rightarrow F^{d}$ is a toplinear isomorphism, there are open convex neighbourhoods $W$ and $W^{\prime}$ of 0 in $E^{d}$ and $F^{d}$ respectively such that $\Phi$ is a $C^{I}$-diffeomorphism of an open neighbourhood of $\bar{W}$ onto an open neighbourhood of $\bar{W}^{\prime}(\bar{W}, \bar{W}$ being the closures of $W, W^{\prime}$ in $E^{d}, F^{d}$ respectively) and $W$ is contained in an open ball centred at 0 in $E^{d}$ and of radius $\rho<\min \left(\delta^{\prime} / 3, \gamma / 3\right)$.

Theorem (2.1) then follows from the following three lemmas.

(2.2). For alz $i \geq d$ and alz $u \in W \cap E^{i}, D \Phi(u): E^{i}+F^{i}$ is a toplinear isomorphism of Banach spaces.

Proof. Using (18) we can proceed as in [3, Lemma 3.1.2].

(2.3). $\Phi\left(W \cap E^{i}\right)=W^{\prime} \cap F^{i}$ for all $i \geq d$.

Proof. For all $y \in W \cap E^{i}$ we have

$$
\Phi(y)=\Phi(y)-\Phi(0)=D \Phi(0) y-\int_{0}^{1}[D \Phi(0) y-D \Phi(t y) y] d t .
$$

Hence, by (14),

$$
|\Phi(y)|_{i} \geq \gamma|y|_{i}-\delta_{i}|y|_{i-1}-\left|\int_{0}^{1}[D \Phi(0) y-D \Phi(t y) y] d t\right|_{i} .
$$

Furthermore, by (17), for all $y \in W \cap E^{i}$, we have 


$$
\left|\int_{0}^{1}[D \Phi(0) y-D \Phi(t y) y] d t\right|_{i} \leq(\gamma / 3)|y|_{i}+D_{i}|y|_{i-1}
$$

Hence, for all $i \geq d+1$ and all $y \in W \cap E^{i}$, we have

$$
|\Phi(y)|_{i} \geq(2 \gamma / 3)|y|_{i}-D_{i}^{\prime}|y|_{i-1} \quad\left(D_{i}^{\prime}=\delta_{i}+D_{i}\right) \text {. }
$$

Using (20) we can proceed just as in [3, Lemma 3.1.3].

(2.4). There is an open neighbourhood $W_{1}^{\prime}$ of 0 in $F^{d}$ such that $W_{1}^{\prime} \subseteq W^{\prime}$, and the following inequalities hold for all $i \geq d+1$, all $u \in W_{1}^{\prime} \cap F^{i}$ and all $v \in F^{i}$,

$$
\begin{aligned}
& \text { (i) }\left|D \Phi^{-1}(u) v\right|_{i} \leq C^{\prime}|v|_{i}+D_{i}^{\prime}|v|_{i-1} \text {, } \\
& \text { (ii) }\left|D \Phi^{-1}(0) v\right|_{i} \geq \gamma^{\prime}|v|_{i}-\delta_{i}^{\prime}|v|_{i-1} \text {, }
\end{aligned}
$$

where $C^{\prime}, \gamma^{\prime}, D_{i}^{\prime}$ and $\delta_{i}^{\prime}$ are positive constants $\left(^{\prime}\right.$ and $\gamma^{\prime}$ being independent of $i$ ).

Proof. Use (18) and (20) and proceed as in [3, Lemma 3.1.4].

REMARK 1. Theorem (2.1) can be stated and proved for any $a \in U$ and $b=\Phi(a)$.

REMARK 2. The inverse map $\Phi^{-1}$ is also a regular $C_{B \Gamma}^{1}$ map.

\section{Application}

In this section, we shall prove, as an application of our Inverse Mapping Theorem, a result of Sunada on the local finite dimensionality of the solution space of a non linear elliptic differential operator with smooth coefficients [6].

Let $M$ be a compact $C^{\infty}$ manifold without boundary, $E, F$ be two finite-dimensional vector bundles over $M$. We denote by $S(E)$ and $S(F)$ the spaces of $C^{\infty}$ secticns of $E$ and $F$ respectively. Let $L: S(E) \rightarrow S(F)$ be a non linear differential operator of order $m$ with smooth coefficients [4], [5]. Suppose that $L$ is eiliptic at $0 \in S(E)$ 
(that is, the linearisation $d_{0} L$ is a linear elliptic operator [2], [4]) and assume that $L(0)=0$. Put

$$
\begin{aligned}
\Sigma_{0} & =\{t \in S(E): L(t)=0\}, \\
T_{0}(\Sigma) & =\left\{u \in S(E): d_{0} L(u)=0\right\} .
\end{aligned}
$$

Endow $(S(E), S(E))$ with the calibration

$$
\Gamma=\left\{\left(\|\cdot\|_{i},\|\cdot\|_{i}\right): i \geq m\right\},
$$

where, for each $i \geq m,\|\|_{i}$ is the $H$-norm defined in [3], p. 28 .

Put $D=\lambda_{0} L_{1}$ and apply the Hodge-Kodaira theory to the linear elliptic operator $D[4],[6]$. We have the direct decompositions

$$
\begin{aligned}
& S(E)=\operatorname{Ker} D \oplus \operatorname{Im} D^{*}=T_{0}(\Sigma) \oplus \operatorname{Im} D^{*}, \\
& S(F)=\operatorname{Ker} D^{*} \oplus \operatorname{Im} D .
\end{aligned}
$$

Let $H: S(E) \rightarrow T_{0}(\Sigma)=\operatorname{Ker} D$ and $K: S(E) \rightarrow \operatorname{Im} D$ be the corresponding projections, and consider the bifurcation operator, [6],

$$
\Phi: S(E) \rightarrow T_{0}(\Sigma) \oplus \operatorname{Im} D
$$

defined by $\Phi(t)=H(t) \oplus K \circ L(t)$ for all $t \in S(E)$, where $T_{0}(\Sigma) \oplus \operatorname{Im} D$ is the topological direct sum of the Fréchet spaces $T_{0}(\Sigma)$ and $\operatorname{Im} D$. Notice that $\operatorname{Im} D$ and $\operatorname{Im} D^{*}$ are closed in $S(F)$ and $S(E)$ respectively, $\operatorname{Ker} D$ and $\operatorname{Ker} D^{*}$ are finite-dimensional, and $\Phi(0)=(0,0)$.

Endow $T_{0}(\Sigma)$ and $\operatorname{Im} D$ with the relative calibrations and define the sum-calibration for $G=T_{0}(\Sigma) \oplus \operatorname{Im} D$,

$$
\Gamma_{G}=\left\{\|\cdot\|_{i}: i \geq m\right\},
$$

where $\|u+v\|_{i}=\|u\|_{i}+\|v\|_{i}$ for all $u+v \in G=T_{0}(\Sigma) \oplus \operatorname{Im} D$.

(3.1). The decompositions (24) and (25) are BГ-direct decompositions that is, the projections are $\beta \Gamma$-continuous linear maps.

Proof. By symmetry, it suffices to prove that (24) is a $B \Gamma$-direct 
decomposition. Let $\square$ denote the restriction of $D D^{*}$ to $\operatorname{Im} D \subseteq S(F)$. Then $\square: \operatorname{Im} D \rightarrow \operatorname{Im} D$ is an isomorphism. Furthermore, for any $\dot{u} \in S(E)$, the element $u-D \square^{-1} D u \in \operatorname{Ker} D$. Thus, the identity $u=\left(u-D^{*} \square^{-1} D u\right)+D^{*} \square^{-1} D u$ shows that the projections corresponding to the decomposition (24) are

$$
H: u \mapsto u-D \square^{-1} D u \text { and } P: u \mapsto D * \square^{-1} D u
$$

It suffices to show that $P$ is a $B \Gamma$-continuous linear map. Now, since $D$ and $D^{*}$ are differential operators of order $m$, we have by [3], p. 73 ,

(28) $\|D u\|_{i} \leq C\|u\|_{i+m}+D_{i}\|u\|_{i+m-1},\left\|D^{*} v\right\|_{i} \leq C^{\prime}\|v\|_{i+m}+D_{i}^{\prime}\|v\|_{i+m-1}$, where $C, C^{\prime}$ and $D_{i}, D_{i}^{\prime}$ are positive constants as usual.

Since $\square$ is the restriction of a linear elliptic differential operator of order $2 m$, [3, Lemma 5.2.1] or [1, p. 358] gives

$$
\left\|\square^{-1} w\right\|_{i} \leq C^{\prime \prime}\|w\|_{i-2 m}+D_{i}^{\prime \prime}\|w\|_{i-2 m-1}
$$

Thus it follows from (28) and (29) that, for all $u$ and all $i \geq m+1$,

$$
\|P u\|_{i} \leq \tilde{C}\|u\|_{i}+\tilde{D}_{i}\|u\|_{i-1} \text {, }
$$

where $\tilde{C}, \tilde{D}_{i}$ are positive constants $(\tilde{C}$ being independent of $i)$.

(3.2). Let $L: S(E) \rightarrow S(F)$ be a (non-linear) differential operator of order $m$ and let $d=\operatorname{dim} M+5$ ( $M$ being the base space of $E$ and $F$ ). Denote by $J^{m} E$ the $m$-jet bundle of $E$ and by $J^{m}: S(E) \rightarrow S\left(J^{m} E\right)$ the m-jet extension. Endow the pair $(S(E), S(F))$ with the calibration

$$
\Gamma=\left\{\left(\|\cdot\|_{m+i},\|\cdot\|_{i}\right\}: i \geq d\right\},
$$

where, for each $j,\|\cdot\|_{j}$ is the $H$-norm defined as above.

Let $W$ be a relatively compact open tubular neighbourhood of the zero section of $J_{E}^{m}, S(W)=\left\{u \in S\left(J^{m} E\right): u(x) \in W\right.$ for alz $\left.x \in M\right\}$, and suppose that $U$ is an open neighbourhood of the zero element in $S(E)$ such that $f^{m}(U) \subseteq S(W)$ and $U=\Omega \cap S(E)$ where $\Omega$ is an open ball 
centred at 0 in $S^{m+d}(E)$, the completion of $S(E)$ with respect to the norm $\|\cdot\|_{m+d}$.

Then $L: U \subseteq S(E)+S(E)$ is a regular $C_{B \tilde{\Gamma}}^{\mathcal{l}}$ map with respect to the calibration $\tilde{\Gamma}=\left\{\left(\|\cdot\|_{m+i},\|\cdot\|_{i}\right\}: i \geq d+1\right\}$ for the pair $(S(E), S(F))$.

Proof. L may be factorised [4], [5], [6] as follows :

$$
S(E) \stackrel{J^{m}}{\longrightarrow} S\left(J^{m} E\right) \stackrel{\Phi}{\longrightarrow} S(F)
$$

where $\Phi=\phi_{*}: S\left(J^{m} E\right) \rightarrow S(F)$ is the induced map of a $C^{\infty}$ fibre bundle morphism $\phi: J_{E}^{m} \rightarrow F$ which is a fortiori a fibre preserving map.

Endow $S\left(J^{m} E\right)$ with the calibration $\left\{\|\cdot\|_{i}: i \geq d\right\}$, where for each $i,\|\cdot\|_{i}$ is the $H$-norm in $S\left(J_{E}^{m}\right)[3$, p. 28].

Since $J^{m}$ is linear continuous, by using the chain rule and [3, Lemma 2.5.3], we have for all $u \in U$, all $v, v_{1}, v_{2} \in S(E)$,

(32) $\|D L(u) v\|_{i}=\left\|(D \Phi) J_{(u)}^{m} J^{m}(v)\right\|_{i}$

$$
\leq C\left\{\left\|J^{m}(u)\right\|_{i}\left\|J^{m}(v)\right\|_{d}+\left\|J^{m}(v)\right\|_{i}\right\}+P_{i}\left(\left\|J^{m}(u)\right\|_{i-1}\right)\left\|J^{m}(v)\right\|_{i-1},
$$

$$
\begin{aligned}
& \left\|D^{2} L(u) v_{1} v_{2}\right\|_{i} \\
& =\left\|\left(D^{2} \Phi\right)_{J^{m}(u)} J^{m}\left(v_{1}\right) J^{m}\left(v_{2}\right)\right\|_{i} \\
& \leq c\left\{\left\|J^{m}(u)\right\|_{i}\left\|J^{m}\left(v_{1}\right)\right\|_{d}\left\|J^{m}\left(v_{2}\right)\right\|_{d}+\left\|J^{m}\left(v_{1}\right)\right\|_{d}\left\|J^{m}\left(v_{2}\right)\right\|_{i}+\left\|J^{m}\left(v_{1}\right)\right\|_{i}\left\|J^{m}\left(v_{2}\right)\right\|_{d}\right\} \\
& +P_{i}\left(\left\|J^{m}(u)\right\|_{i-1}\right)\left\|J^{m}\left(v_{1}\right)\right\|_{i-1}\left\|J^{m}\left(v_{2}\right)\right\|_{i-1} \text {. }
\end{aligned}
$$

Hence, using [3, Lemma 5.1.2], p. 70, it is easily seen that $L$ satisfies the hypotheses of (1.2) and (3.2) is proved.

Now we can prove the following theorem which is due to Sunada [6, Theorem 2]. 
(3.3). I maps a neighbourhood of 0 in $S(E)$ diffeomorphically onto a neighbourhood of $\Phi(0)=(0,0)$ in $T_{0}(\Sigma) \oplus \operatorname{Im} D$.

Proof. Endow $\left(S(E), T_{0}(\Sigma) \oplus \operatorname{Im} D\right)$ with the calibration

$$
\Gamma=\left\{\left(\|\cdot\|_{m+i},\|\cdot\|_{i}\right): i \geq d\right\}
$$

where the second norm, $\|\cdot\|_{i}$, is given in (27).

Then, in view of (3.1) and (3.2), we may suppose that $\Phi: U=\Omega \cap S(E) \subseteq S(E) \rightarrow G=T_{0}(\Sigma) \oplus \operatorname{Im} D$ is a regular $C_{\beta \Gamma}^{l}$ map (recalibrate if needed).

Furthermore, it follows quickly that

$$
D \Phi(0)=H \oplus \operatorname{Kod}_{0} L=H \oplus K \circ D=H \oplus D \text {. }
$$

From this it is easily seen that $D \Phi(0): S(E) \rightarrow G$ is a toplinear isomorphism [6, Lemma 5$]$.

Now, since $D$ is a linear elliptic operator of order $m,[1]$ or [3, Lemma 5.2.1] gives us, for all $u$ and all $i \geq d+1$,

$$
\|D u\|_{i} \geq \gamma\|u\|_{m+i}-\delta_{i}\|u\|_{m+i-1}
$$

where $\gamma$ and $\delta_{i}$ are positive constants $(\gamma$ being independent of $i$ ). Thus, for all $u \in S(E)$ and all $i \geq d+1$, we have, by (35),

$$
\begin{aligned}
\|D \Phi(0) u\|_{i} & =\|H(u)\|_{i}+\|D u\|_{i} \geq\|D u\|_{i} \\
& \geq \gamma\|u\|_{m+i}-\delta_{i}\|u\|_{m+i-1} .
\end{aligned}
$$

Therefore $D \Phi(0) \in \mathrm{GL}_{B \Gamma}(S(E), G)$ and (3.3) then follows from (2.1).

It is an easy consequence of (3.3) that if $E, F$ are two $C^{\infty}$ fibre bundles over $M$ and if $L: S(E) \rightarrow S(F)$ is a non-linear differential operator with smooth coefficients and is elliptic at $s \in S(E)$, [6], then the solution space $\Sigma_{s}=\{t \in S(E): L(t)=L(s)\}$ is locally a finitedimensional subset in $S(E)$ near $s \quad[6$, Theorem 1$]$. 


\section{References}

[1] M. Kuranishi, "A new proof for regularity of solutions of elliptic differential operators", Global analysis and its applications, Vol. II, 355-361 (Lectures, Internat. Sem. Course, Internat. Centre Theoret. Phys., Trieste, 1972. International Atomic Energy Agency, Vienna, 1974).

[2] Reghavan Narasimhan, Analysis on real and complex manifolds (Advanced Studies in Pure Mathematics, 1. Masson, Paris; North-Holland, Amsterdam; 1973).

[3] Hideki Omori, Infinite dimensional Lie transformation groups (Lecture Notes in Mathematics, 427. Springer-Verlag, Berlin, Heidelberg, New York, 1974).

[4] R.S. Palais, "Differential operators on vector bundles", Seminar on the Atiyah-Singer index theorem, 51-93 (Princeton University Press, Princeton, New Jersey, 1965).

[5] Richard S. Palais, Foundation of global non-linear analysis (Benjamin, New York, Amsterdam, 1968).

[6] Toshikazu Sunada, "Non-linear elliptic operators on a compact manifold and an implicit function theorem", Nagoya Math. J. 56 (1974), $175-200$.

[7] Sadayuki Yamamuro, A theory of differentiation in locally convex spaces (Memoirs of the American Mathematical Society, 212. American Mathematical Society, Providence, Rhode Island, 1979).

[8] Sadayuki Yamamuro, "A note on Omori-Lie groups", Bull. Austral. Math. Soc. $19(1978), 333-349$.

Department of Pure Mathematics, University of Sydney, Sydney, New South Wales 2006, Australia. 